\title{
Early and late outcomes of acute type A aortic dissection with intramural hematoma
}

\author{
Anthony L. Estrera, MD, FACS, Harleen K. Sandhu, MD, MPH, Samuel S. Leake, BS, \\ Kristofer M. Charlton-Ouw, MD, FACS, Rana O. Afifi, MD, Charles C. Miller III, PhD, and \\ Hazim J. Safi, MD, FACS
} Introduction: Controversy remains regarding management of acute type A dissection with intramural
hematoma (IMH). Our purpose was to analyze our experience and report outcomes after repair of acute type
A aortic dissection with IMH.

\begin{abstract}
Methods: We analyzed all patients from a single center who underwent open repair for acute type A aortic dissection with IMH.

Results: Between 2000 and 2013, we performed 418 repairs for acute type A aortic dissection. These were divided into 2 groups of patients: 64 patients $(15 \%)$ with type A IMH and 354 patients $(85 \%)$ with typical dissection. Those with IMH were older $(62.4 \pm 13.9$ years vs $56.7 \pm 14.7$ years; $P<.0046)$ and presented with reduced renal function (ie, glomerular filtration rate) $(P<.0341)$, less frequently with distal malperfusion, and less frequently with rupture $(P<.0116)$. With IMH, the time from presentation to repair was, by strategy, longer (median, 67 vs 6 hours; $P<.0001$ ), but no mortality occurred within 3 days of presentation. Mortality with IMH did not differ from typical dissection: 7 out of 64 patients $(10.9 \%)$ versus 52 out of 354 patients $(14.7 \% ; P=.4276)$. A lower incidence of postoperative dialysis in the IMH group approached significance: 6 out of 63 patients $(9.5 \%)$ versus 64 out of 347 patients $(18.4 \% ; P=.0820)$. When adjusted for age and renal function, late survival was improved with IMH $(P<.0343)$.
\end{abstract}

Conclusions: Repair of acute type A aortic dissection with IMH is associated with significant early morbidity and mortality, differing minimally from typical aortic dissection. Although expectant repair within 3 days may be applied, the purposeful delay imparted little advantage. Improved late outcomes may be seen with IMH, but continued long-term surveillance is required for verification. (J Thorac Cardiovasc Surg 2015;149:137-42)

See related commentary on page 143 .

Intramural hematoma (IMH), first described as "aortic dissection without intimal flap" by Krukenberg in $1920,{ }^{1}$ remains a therapeutic challenge. It is generally agreed that the management of descending thoracic, or Stanford type $\mathrm{B}$, IMH is primarily medical, but the optimal treatment of ascending, or Stanford type A, IMH remains unclear. ${ }^{2-7}$

\footnotetext{
From the Department of Cardiothoracic and Vascular Surgery, Clinical Science Program, The University of Texas Medical School at Houston, and Memorial Hermann Hospital, Houston, Tex.

Disclosures: Authors have nothing to disclose with regard to commercial support.

Read at The American Association for Thoracic Surgery Aortic Symposium,

New York, New York, April 24-25, 2014.

Received for publication May 14, 2014; revisions received Sept 18, 2014; accepted for publication Oct 5, 2014.

Address for reprints: Anthony L. Estrera, MD, FACS, Department of Cardiothoracic and Vascular Surgery, The University of Texas Medical School at Houston, 6400 Fannin St, Ste 2850, Houston, TX 77030 (E-mail: Anthony.L.Estrera@uth. tmc.edu).

0022-5223/\$0.00

Published by Elsevier Inc. on behalf of The American Association for Thoracic Surgery

http://dx.doi.org/10.1016/j.jtcvs.2014.10.028
}

Recent guidelines from the Society of Thoracic Surgeons recommend that type A IMH be "reasonably treated with surgical intervention" and that medical management only be applied to patients considered too high risk for open repair, under the conditions that they remain asymptomatic and radiographically stable. ${ }^{8}$ However, some Asian series have demonstrated excellent early outcomes with medical management alone. ${ }^{9-12}$

We previously published our experience with acute type A IMH in 36 patients who underwent immediate surgical repair, delayed (expectant) repair, and medical management. ${ }^{13}$ In this analysis, we also analyzed the hazard of conversion of the IMH to typical dissection in relation to time of presentation. We identified that $33 \%$ of cases converted to typical dissection by the time of open repair and that the risk of conversion was greatest at 8 days. Considering that the risk was low for early conversion, we adopted an approach to perform delayed open repair of acute type A IMH within 3 days of presentation, with the reasoning that delayed management might impart an advantage, as had been observed with traumatic aortic injury. ${ }^{14}$ Thus, the purpose of our study was to analyze our experience with open repair for acute type A IMH and report early and late outcomes. 


\section{Abbreviations and Acronyms \\ GFR $=$ glomerular infiltration rate \\ $\mathrm{IMH}=$ intramural hematoma}

\section{METHODS}

The Committee for Protection of Human Subjects for the University of Texas Medical School at Houston, the local institutional review board, approved this study and consent was waived.

\section{Definitions}

Type A IMH was defined as aortic dissection without identifiable intimal tear and lack of flow in the false lumen of the ascending aorta. ${ }^{15}$ Identification of type A IMH was first obtained by computed tomography and confirmation that a dissection flap was not present was made by transesophageal echocardiography. Patients with identifiable flow in the false lumen of the descending and thoracoabdominal aorta were included for analysis. Acute dissection was defined as dissection occurring within 2 weeks of presentation. Conversion to typical dissection was determined at either operative examination or radiographic evaluation before surgery. The time of the initial onset of pain (in hours) determined the onset of IMH. Time of symptom onset is defined by the time identified by patient history as the first instance of symptoms. Time to operative repair was defined as time from onset of pain to operative repair. Typical dissection was dissection with any free flow into the false channel. Early mortality included in-hospital death and death occurring within 30 days of surgery. Stroke was defined as any gross focal neurologic brain injury, either temporary or permanent, identified on neurologic examination by a neurology consultant and confirmed with computed tomography or magnetic resonance imaging. The glomerular filtration rate (GFR) was calculated by the Cockcroft-Gault method. ${ }^{16}$ Respiratory dysfunction was defined as prolonged intubation $>24$ hours, reintubation, or need for tracheostomy. Reoperation was defined as the need for any surgical procedure, including re-exploration for bleeding, an abdominal procedure, tracheostomy, or an aortovascular procedure. Hypotension was defined as systolic blood pressure $<90 \mathrm{~mm} \mathrm{Hg}$, significant aortic insufficiency was defined as moderate to severe grade, and aortic rupture was defined as the presence of blood in the pericardial sac with tamponade. Malperfusion syndrome included any clinically evident organ malperfusion; for example, cerebral, spinal, visceral, renal, and limb.

\section{Surgical Approach}

All cases of typical dissection underwent urgent or emergent repair unless contraindicated for neurologic devastation, metastatic cancer, or patient refusal. Nonoperative cases were excluded. For patients with $\mathrm{IMH}$, operative repair was performed emergently for patients in extremis and expectantly (eventual) in patients whose symptoms were controlled and whose vital signs were stable. Surgical management used cardiopulmonary bypass, deep hypothermic circulatory arrest, and retrograde cerebral perfusion, as described previously. ${ }^{17}$ Cardiopulmonary bypass was routinely initiated via femoral or axillary artery cannulation and axillary artery cannulation was used in cases of femoral artery pulse deficit. Adequate arterial inflow perfusion was confirmed by monitoring cerebral oximetry and appropriate decreases in nasopharyngeal and bladder temperatures. Venous cannulation was via the superior and inferior vena cava. Both antegrade and retrograde cold blood cardioplegia provided myocardial protection. A left ventricular sump graft catheter was inserted into the right superior pulmonary vein. Once adequate cooling was achieved, cardiopulmonary bypass was discontinued and the circulation was arrested. Retrograde cerebral perfusion was started via the superior vena cava cannula. The patient was placed in slight
Trendelenburg position. The ascending aorta was then opened and inspected. If a tear was identified, it was resected. The arch was replaced only if enlarged $>5 \mathrm{~cm}$; partial arch replacement (hemiarch) with an open distal anastomosis was performed otherwise. Any remaining thrombus within the wall was removed before performing the anastomosis. Once the anastomosis was completed, the graft was deaired and clamped, and antegrade perfusion was initiated via a sidearm branch of the aortic graft. After systemic warming was begun, the proximal reconstruction was performed. The remaining ascending aorta was resected to the sinotubular junction. If the aortic valve was intact and the aortic root was not enlarged $>5 \mathrm{~cm}$ or compromised, the proximal anastomosis was performed after resuspension of the aortic valve.

\section{Statistical Methods}

Data were collected from chart reviews done by a trained nurse evaluator and were entered into a dedicated Microsoft Access database (Microsoft Corp, Redmond, Wash). Analysis was retrospective. Patient follow-up was obtained by direct patient contact, telephone interview, or the National Death Index and was complete for mortality and interventions. Data were managed under Health Insurance Portability and Accountability Act confidentiality guidelines in an Access database with encrypted patient identifiers.

Normally distributed continuous variables were compared by unpaired $t$ test and are presented as mean \pm standard deviation. Transfusion products, which arise from a zero-inflated skewed distribution, and other continuous variables not meeting normal assumptions are presented as median and interquartile range. Statistical tests for nonnormal continuous data were conducted by Wilcoxon rank-sum procedures. Dichotomous variables were analyzed by contingency tables methods, with $\chi^{2} P$ values reported unless any expected cell value was less than 5 , in which case Fisher exact $P$ value is reported. Mortality hazard for the first 30 days (Figure 1) was computed using life table methods. Hazard was defined as the instantaneous probability of occurrence for each life table interval. ${ }^{18}$ Long-term (10-year) (Figure 2) mortality predictors were identified using Cox proportional-hazards regression analysis. A formal test of the long-term proportional hazards assumption was performed. Figure 2 represents direct-adjusted survival functions from a Cox model, including terms for IMH, estimated GFR, and age. All computations were performed using SAS version 9.3 (SAS Institute Inc, Cary, NC). Long-term survival was compared between groups using Kaplan-Meier analysis.

\section{RESULTS \\ Patients}

Between 2000 and 2013, we performed 418 repairs for acute type A aortic dissection. Of this cohort, 15\% (64 out of 418 patients) had associated IMH and $85 \%$ (354 out of 418 patients) had typical type A dissection. Patients with IMH were older than those with typical dissection $(62.4 \pm 13.9$ vs $56.7 \pm 14.7$ years $)$, with slightly more women $(37.5 \%$ [ 24 out of 64 patients] vs $26.8 \%$ [ 95 out of 354 patients]; $P=.0819$ ). Patients with IMH presented less frequently with shortness of breath, malperfusion syndromes, pulse deficits, significant aortic insufficiency, hypotension, and rupture (Table 1). Cannulation strategy did not differ between IMH and typical dissection nor did aortic root replacement or complete transverse arch replacement (Table 1). In the IMH group, cardiopulmonary bypass time, aortic crossclamp time, and retrograde cerebral perfusion times were shorter when compared with the typical type A repair group (Table 2). Intraoperative blood use did not differ between groups (Table 2). 


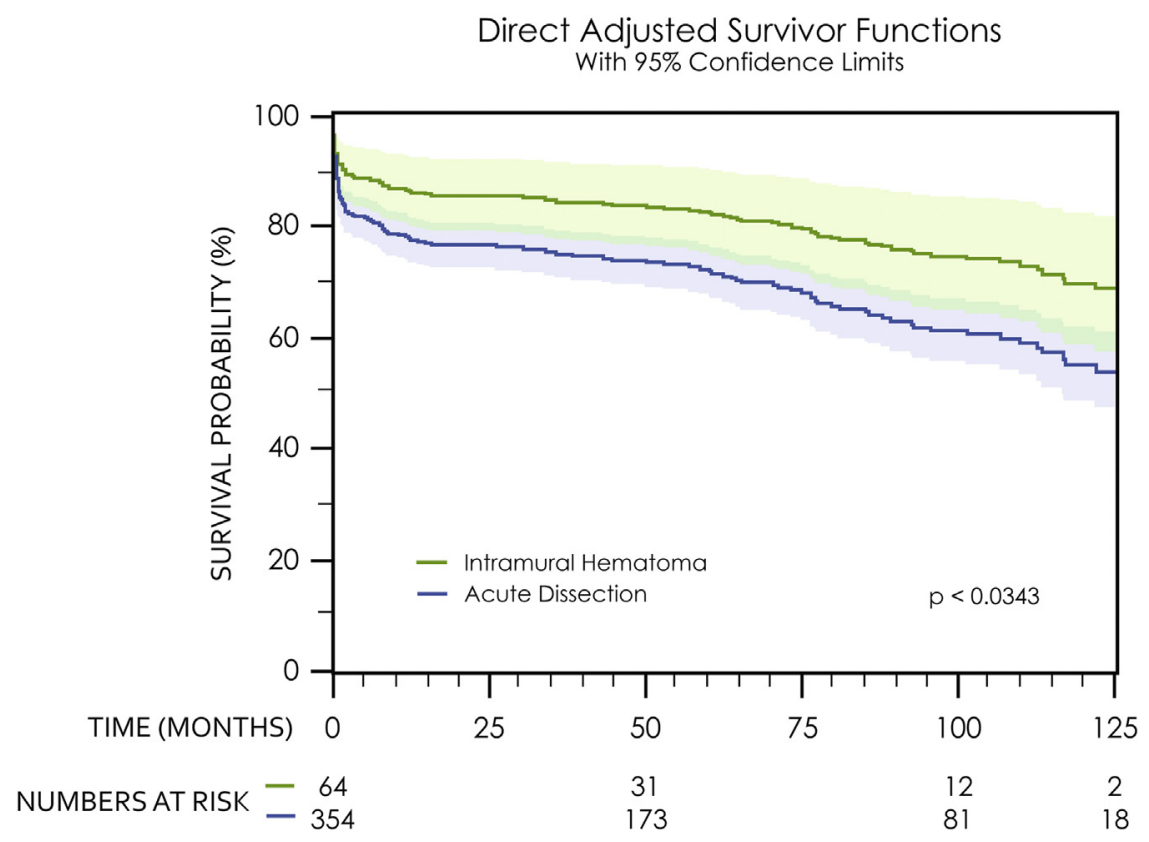

FIGURE 1. Kaplan-Meier estimate for late survival adjusted for age and preoperative renal dysfunction of patients operated upon for acute type A intramural hematoma compared with typical or classic type A aortic dissection.

\section{Early Mortality and Morbidity}

Early mortality with acute type A IMH did not differ from typical acute type A dissection, 10.9\% (7 out of 64 patients) versus $14.7 \%$ (52 out of 354 patients) $(P=.4322)$, respectively. There were 28 deaths during the first 3 days in the acute dissection group and none in the same time period for IMH. Reoperation for bleeding, any reoperation, respiratory dysfunction, stroke, and renal dysfunction were not significantly different between IMH and typical dissection groups. The lower incidence of postoperative dialysis in the IMH group approached significance: 6 out of 63 patients $(9.5 \%)$ versus 64 out of 347 patients $(18.4 \%)$ $(P=.0820)($ Table 3$)$.

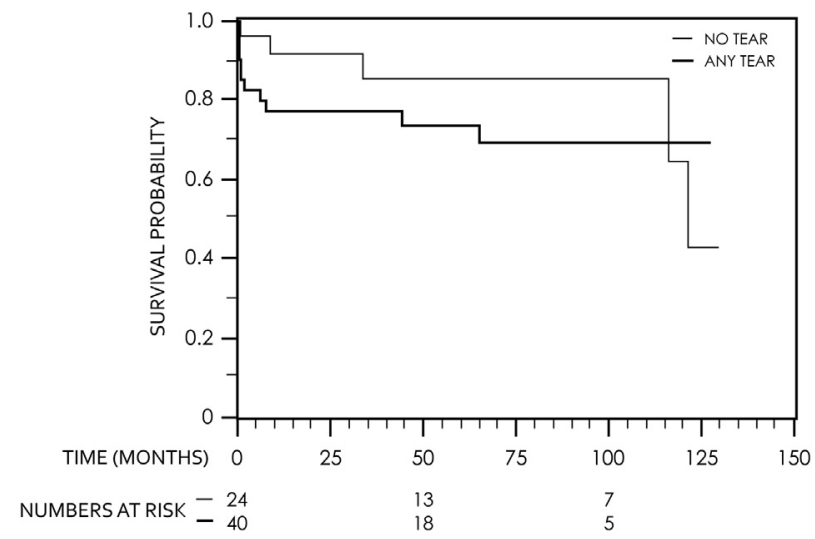

FIGURE 2. Kaplan-Meier estimate for late survival of repaired type A intramural hematoma comparing no tear versus tear in the remaining thoracic aorta.
Time from presentation to surgical repair was longer with IMH than typical dissection-median 67 versus 6 hours- $(P<.0001)$, as was expected with the implementation of the delayed, eventual repair, with IMH no mortality occurring within 3 days of presentation. In contrast, 28 deaths occurred within 3 days of presentation in the typical type A dissection cohort. The hazard function (ie, instantaneous risk for mortality) was not significantly different between type A IMH and typical dissection $(P=.2356)$ (Figure 1). Notably, an increase in hazard mortality was observed at 6 days in patients with type A IMH.

\section{Aortic Characteristics}

Mean aortic diameter was similar in patients with type A IMH compared with typical dissection: $4.95 \pm 0.76 \mathrm{~cm}$ versus $5.11 \pm 1.15 \mathrm{~cm}(P<.1691)$. Before operative repair, of patients with type A IMH, tears in the ascending aorta were identified in $39 \%$ of patients ( 25 out of 64 ), the transverse arch in $3 \%$ of patients ( 2 out of 64 ), and descending thoracic aorta in $23 \%$ of patients (15 out of 64). In $34 \%$ of patients ( 22 out of 64 ) with type A IMH, no aortic tear was identified at the time of surgery. After operative repair of the ascending aorta, a distinguishable flap was identified in the distal aorta (descending or thoracoabdominal aorta) in $38 \%$ of patients ( 25 out of 64). Three cases (5\%) involved IMH limited to the ascending aorta only (DeBakey type 2).

Of the early survivors $(n=57)$ during follow-up, favorable remodeling (ie, reduction or complete resolution of the distal aortic IMH) was observed in $26 \%$ of patients (15 out of 57). Patients with unfavorable distal remodeling 
TABLE 1. Preoperative variables for acute type A intramural hematoma

\begin{tabular}{lccc}
\hline \multicolumn{1}{c}{ Variable } & $\begin{array}{c}\text { IMH } \\
(\mathbf{n}=\mathbf{6 4})\end{array}$ & $\begin{array}{c}\text { Acute dissection } \\
(\mathbf{n = 3 5 4 )}\end{array}$ & $\begin{array}{c}\boldsymbol{P} \\
\text { value }\end{array}$ \\
\hline Female & $24 / 64(37.5 \%)$ & $95 / 354(26.8 \%)$ & .0819 \\
Age & $62.4 \pm 13.9$ & $56.7 \pm 14.7$ & .0046 \\
Estimated glomerular & $79.8 \pm 35.0$ & $90.8 \pm 45.8$ & .0341 \\
$\quad$ filtration rate* & & & \\
Chest pain & $56 / 64(87.5 \%)$ & $303 / 354(85.6 \%)$ & .6868 \\
Shortness of breath & $5 / 64(7.8 \%)$ & $79 / 354(22.3 \%)$ & .0077 \\
Any malperfusion syndrome & $14 / 64(21.9 \%)$ & $127 / 354(35.8 \%)$ & .0292 \\
Leg ischemia & $1 / 64(1.6 \%)$ & $59 / 354(16.6 \%)$ & .0015 \\
Pulse deficit & $0 / 64(0.0 \%)$ & $44 / 354(12.4 \%)$ & .0029 \\
Stroke on admission & $4 / 64(6.3 \%)$ & $28 / 354(7.9 \%)$ & .6499 \\
Renal malperfusion & $2 / 64(3.1 \%)$ & $20 / 354(5.6 \%)$ & .4052 \\
Hypotension & $11 / 64(17.2 \%)$ & $112 / 354(31.6 \%)$ & .0197 \\
Aortic insufficiency & $24 / 64(37.5 \%)$ & $234 / 354(66.2 \%)$ & .0001 \\
Rupture & $3 / 64(4.7 \%)$ & $60 / 354(16.9 \%)$ & .0116 \\
\hline
\end{tabular}

$I M H$, Intramural hematoma. *Cockcroft-Gault estimated glomerular filtration rate (milliliters/minute) $1.73 \mathrm{~m}^{2}$.

were noted to have tears in either the ascending, arch, or descending thoracic aorta in $59 \%$ of patients ( 25 out of 42 ).

\section{Late Outcomes}

Median follow-up was 48.8 months. Unadjusted late survival between type A IMH and typical type A repair did not differ significantly. However, when adjusted for age and renal function, 1-, 5- and 10-year survival for type A IMH was $86.3 \%, 82.6 \%$, and $69.7 \%$, respectively, which was significantly better than survival of typical type A dissection: $77.7 \%, 72.3 \%$, and $55 \%$, respectively $(P<.0343)$ (Figure 2). Analyzing the presence and location of the tear in relation to late survival, the presence of a tear in the ascending aorta identified at the time of open repair of type A IMH repair was not associated with worse late survival (Figure 3). Similarly, patients with repaired type A IMH in which no tear was identified did not demonstrate improved late survival. In patients with favorable remodeling, no late distal reinterventions occurred ( 0 out of 15 patients). No difference in reintervention was noted in patients with unfavorable distal remodeling $(7 \% ; 3$ out of 42 patients) compared with favorable remodeling $(P=.7847)$. The late reinterventions that occurred after repair of type A IMH repair included thoracic endovascular aortic repair for descending thoracic aneurysm at 3 months, open thoracoabdominal aortic aneurysm (extent 1 ) repair at 6 months, and thoracic endovascular aortic repair for descending thoracic aneurysm at 22 months. One patient required proximal aortic root and transverse arch replacement at 11 months from initial type A IMH repair.

Long-term (10-year) mortality was significantly associated with increasing age (hazard ratio [HR], 1.04/year; $P<.0001)$, baseline GFR $(<60 ; \mathrm{HR}, 1.64$; $P<.01$ ), and presence of IMH (HR, 0.57; $P<.0343$ ) in multiple Cox regression. A test of the proportional hazards assumption showed no significant departure from proportionality over the 10-year follow-up period because the survival distributions took on a typical declining exponential form.

\section{DISCUSSION}

The precise mechanism that leads to aortic dissection remains unknown, but it is likely that it is related to the

TABLE 2. Intraoperative variables

\begin{tabular}{|c|c|c|c|}
\hline Variable & Intramural hematoma $(n=64)$ & Acute dissection $(n=354)$ & $P$ value \\
\hline Mean aortic diameter, $\mathrm{cm}$ & $4.95 \pm 0.76$ & $5.11 \pm 1.15$ & .1691 \\
\hline Hours from presentation to repair & 67 (IQR 15-110) & 6 (IQR 2-16) & .001 \\
\hline Coronary bypass grafting & $5(7.8 \%)$ & $31(8.7 \%)$ & .0890 \\
\hline Aortic valve resuspension & $57(89.1 \%)$ & $300(84.8 \%)$ & .3722 \\
\hline Aortic root reconstruction & $52(81.3 \%)$ & $268(75.5 \%)$ & .3183 \\
\hline Total arch replacement & $5(8 \%)$ & $32(9 \%)$ & .9795 \\
\hline \multicolumn{4}{|l|}{ Cannulation } \\
\hline Femoral & 50 & 308 & .22 \\
\hline Axillary & 2 & 16 & 1.00 \\
\hline Ascending & 12 & 29 & .13 \\
\hline Cardiopulmonary bypass time, $\min$ & $148 \pm 55$ & $164 \pm 52$ & .0209 \\
\hline Aortic crossclamp time, min & $92 \pm 37$ & $103 \pm 33$ & .0133 \\
\hline Retrograde cerebral perfusion, min & $25 \pm 11$ & $28 \pm 10$ & .0409 \\
\hline Transfusion with packed red blood cells, units & $* 0=54(\mathrm{IQR} 2-5)$ & $* 0=715(\mathrm{IQR} 3-7)$ & .3374 \\
\hline Transfusion with fresh frozen plasma, units & $* 0=64(\mathrm{IQR} 3-6)$ & $* 0=856(\mathrm{IQR} 3-8)$ & .0530 \\
\hline Transfusion (platelets), units & $* 0=66($ IQR $6-12)$ & $* 0=9312(\mathrm{IQR} 6-16)$ & .1076 \\
\hline Transfusion (platelets), units & $* 0=66($ IQR $6-12)$ & $* 0=9312(\mathrm{IQR} 6-16)$ & .1076 \\
\hline Prior sternotomy & $11 / 64(17.2 \%)$ & $91 / 354(25.6 \%)$ & .1473 \\
\hline Exploratory laparotomy & $5 / 64(7.8 \%)$ & $24 / 354(6.8 \%)$ & .7602 \\
\hline
\end{tabular}

$I Q R$, Interquartile range. *Transfusions arise from a 0 -inflated, skewed distribution. $0=\mathrm{x}$ for each transfusion product is the number of patients who received 0 products. To the immediate right of those figures are the median and the interquartile range for the nonzero values. 
TABLE 3. In-hospital postoperative complications

\begin{tabular}{lccc}
\hline \multicolumn{1}{c}{ Variable } & $\begin{array}{c}\text { Intramural } \\
\text { hematoma } \\
(\mathbf{n = 6 4 )}\end{array}$ & $\begin{array}{c}\text { Acute } \\
\text { dissection } \\
(\mathbf{n}=\mathbf{3 5 4})\end{array}$ & $\begin{array}{c}\boldsymbol{P} \\
\text { value }\end{array}$ \\
\hline Reoperation for bleeding & $3(4.6)$ & $21(5.9)$ & 1.00 \\
Any reoperation* & $11(17.2)$ & $91(25.6)$ & .1442 \\
Postoperative myocardial infarction & $1(1.6)$ & $4(1.1)$ & .7696 \\
Postoperative respiratory dysfunction & $33(51.6)$ & $213(60.0)$ & .1979 \\
Postoperative mesenteric ischemia & $2(3.1)$ & $14(3.9)$ & .7502 \\
Postoperative stroke & $0(0)$ & $4(1.1)$ & .3928 \\
Postoperative dialysis $\dagger$ & $6(9.5)$ & $64(18.5)$ & .0820 \\
Permanent dialysis $\dagger$ & $1(1.6)$ & $31(8.9)$ & .0451 \\
30-d and in-hospital mortality & $7(10.9)$ & $52(14.7)$ & .4276 \\
\hline
\end{tabular}

Values are presented as $\mathrm{n}(\%)$. * In-hospital reinterventions in which no actuarial estimates are necessary. †Nine patients were admitted on dialysis and were not evaluable for postoperative dialysis.

integrity of the aortic wall and the stress forces applied to the wall. It has been well established that the media of the aortic wall plays an integral part in the pathogenesis of aortic aneurysms and dissection and recent data suggest a role of the vasa vasorum that may differ from previously held views. ${ }^{19,20}$ It had been theorized that the vasa vasorum was responsible for IMH, with elevated pressures in the vasa vasorum leading to rupture within the aortic wall. Subsequently, progression and eventual rupture into the intima might occur, leading to typical dissection, ${ }^{3,5,21,22}$ in which case the IMH would be a precursor to acute aortic dissection. ${ }^{4}$ However, this mechanism is unlikely because pressure in the vasa vasorum is not significantly greater than systemic pressure in the intravascular space. Recent studies examining the vasa vasorum have suggested that hyperplasia leading to chronic occlusive disease within the aortic wall leads to chronic medial ischemia and degeneration. ${ }^{19}$ In addition, occlusion of the vasa vasorum may lead to outer medial

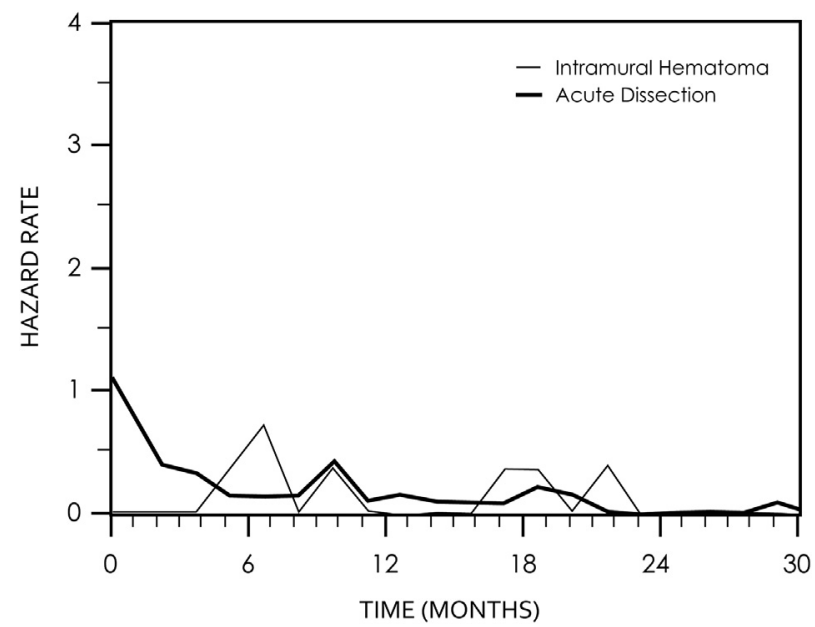

FIGURE 3. Hazard function for early mortality comparing type A intramural hematoma and typical aortic dissection. ischemia and hematoma and dissection, as was observed in aortic specimens in which the tear was identified in the outer one-third of the media. ${ }^{20}$

Much controversy remains regarding acute type A IMH because differences in presentation, diagnosis, risk for progression, and outcomes appear dependent on geographic location and, possibly, on ethnicity. Previous reports have demonstrated obvious differences between Asian and Western cohorts. The Asian series report a greater incidence of type A IMH and the patients are more frequently women, at greater risk for progression to typical dissection with associated complications, and have a lower mortality when managed medically. ${ }^{4,-12}$ In contrast, many Western reports maintain that type A IMH acts similarly to typical dissection and, thus, they recommend a more aggressive stance. ${ }^{6,7,13,23}$ The reasons for these differences between geographic location or by ethnicity remain unclear, although some have suggested that aggressive radiologic surveillance programs may explain the higher incidence of IMH in Asian cohorts. ${ }^{5}$

The management of acute type A aortic dissection with IMH also remains controversial because it appears that pathology may vary with geographic location. In several Asian reports, patients with IMH meeting specific criteria were managed medically with good results. In those reports, certain risk factors were identified that suggested a greater risk for late complications, such as aortic size $>5.0 \mathrm{~cm}$ and, in some reports, thickness of the aortic wall, from 1.2 to $1.6 \mathrm{~cm}^{11,12}$ However, late complications and conversions to typical acute type A dissection may occur in up to $30 \%$ to $40 \%$ of cases in these series.

In our previous report, ${ }^{13}$ we examined early outcomes of a selective, delayed (eventual) approach to the treatment of type A IMH. That report analyzed the temporal differences in open management, specifically examining early outcomes and the development of progression or conversion of type A IMH to typical dissection. Because the data suggest that conversion did not occur within 3 days, a delayed approach was adopted based on the hypothesis that delay might allow the ensuing inflammatory response to subside, allow the tissues to become more manageable, and facilitate the subsequent repair. After that report, we adopted an approach of eventual repair in 3 to 4 days for patients with acute type A IMH who remained stable with no signs of progression, with a mean time to repair of 80 hours. Cautions to bear in mind when evaluating the hazards associated with time of symptom onset include patient recall of first symptoms, which may not be reliable, and that patients who died in the field or otherwise had undiagnosed dissection will bias the estimates to some degree because the true population denominator is not known. This limitation is true for any high-mortality condition, such as major trauma, and creates some uncertainty around the estimates. The results of this 
approach demonstrated no difference in early or late outcomes compared with those obtained by the standard, immediate surgical management of typical type A dissection. Because delayed surgery conferred no advantage, yet recognizing that no early ( $<3$ days from presentation) rupture occurred when patients were clinically stable, we currently intervene urgently, as opposed to an emergent fashion, for acute type A IMH. Adoption of this approach requires that patients are managed in an intensive care unit by an aortic surgical service and that effective anti-impulse therapy is achieved.

Late survival for the cohort with typical acute type A aortic dissection did not differ from that of the type A IMH cohort until adjustments for age and preoperative renal dysfunction were made. These adjustments were appropriate, because patients with IMH were older and had a higher incidence of preoperative renal dysfunction. Improved late survival with IMH has been noted in other reports $^{24,25}$ and may reflect the potential for favorable distal remodeling conferred by IMH. Interestingly, the presence or location of an intimal tear did not correlate with late survival in the type A IMH group.

\section{CONCLUSIONS}

Repair of acute type A aortic dissection with IMH is associated with significant early morbidity and mortality, similar to those obtained with typical aortic dissection. Although eventual repair within 3 days may be applied, the purposeful delay appears to impart no clinical advantage. There is reason to expect better late outcomes with type A IMH compared with typical type A dissection, and ongoing long-term surveillance of our patients may verify that point.

The authors thank Chris Akers for his support with medical illustrations, and Jennifer Goodrich, RN, and Fernando Kubrusly, $\mathrm{MD}$, for research support in the department's Clinical Research Program.

\section{References}

1. Krukenberg E. Beiträgezur Frage des Aneurysma dissecans. Beitr Patho Anat Allg Pathol. 1920;67:329-51.

2. Robbins RC, McManus RP, Mitchell RS, Latter DR, Moon MR, Olinger GN, et al. Management of patients with intramural hematoma of the thoracic aorta. Circulation. 1993:88:II1-10.

3. Vilacosta I, San Roman JA, Ferreiros J, Aragoncillo P, Mendez R, Castillo JA, et al. Natural history and serial morphology of aortic intramural hematoma: a novel variant of aortic dissection. Am Heart J. 1997;134:495-507.
4. Yamada T, Tada S, Harada J. Aortic dissection without intimal rupture: diagnosis with MR imaging and CT. Radiology. 1988;168:347-52.

5. Sundt TM. Intramural hematoma and penetrating atherosclerotic ulcer of the aorta. Ann Thorac Surg. 2007;83:S835-41; discussion S46-50.

6. Evangelista A, Mukherjee D, Mehta RH. International Registry of Aortic Dissection Investigators. Acute intramural hematoma of the aorta: a mystery in evolution. Circulation. 2005;111:1063-70.

7. von Kodolitsch Y, Csosz SK, Koschyk DH, Schalwat I, Loose R, Karck M, et al. Intramural hematoma of the aorta: predictors of progression to dissection and rupture. Circulation. 2003;107:1158-63.

8. Svensson LG, Adams DH, Bonow RO, Kouchoukos NT, Miller DC, O'Gara PT, et al. Aortic valve and ascending aorta guidelines for management and quality measures: executive summary. Ann Thorac Surg. 2013;95:1491-505.

9. Moizumi Y, Komatsu T, Motoyoshi N, Tabayashi K. Management of patients with intramural hematoma involving the ascending aorta. J Thorac Cardiovasc Surg. 2002;124:918-24.

10. Kan CB, Chang RY, Chang JP. Optimal initial treatment and clinical outcome of type A aortic intramural hematoma: a clinical review. Eur J Cardiothorac Surg. 2008:33:1002-6.

11. Kitai T, Kaji S, Yamamuro A, Tani T, Tamita K, Kinoshita M, et al. Clinical outcomes of medical therapy and timely operation in initially diagnosed type a aortic intramural hematoma: a 20-year experience. Circulation. 2009; 120(Suppl):S292-8.

12. Song JK, Yim JH, Ahn JM, Kim DH, Kang JW, Lee TY, et al. Outcomes of patients with acute type a aortic intramural hematoma. Circulation. 2009;120: 2046-52.

13. Estrera A, Miller CC 3rd, Lee TY, De Rango P, Abdullah S, Walkes JC, et al. Acute type A intramural hematoma: analysis of current management strategy. Circulation. 2009; 120:S287-91.

14. Estrera AL, Miller CC 3rd, Guajardo-Salinas G, Coogan S, Charlton-Ouw K, Safi HJ, et al. Update on blunt thoracic aortic injury: fifteen-year single-institution experience. J Thorac Cardiovasc Surg. 2013;145(Suppl): S154-8.

15. Song JK. Diagnosis of aortic intramural haematoma. Heart. 2004:90:368-71.

16. Cockcroft DW, Gault MH. Prediction of creatinine clearance from serum creatinine. Nephron. 1976;16:31-41.

17. Safi HJ, Miller CC 3rd, Lee TY, Estrera AL. Repair of ascending and transverse aortic arch. J Thorac Cardiovasc Surg. 2011;142:630-3.

18. Kalbfleisch JD. The statistical analysis of failure time data. New York: John Wiley \& Sons; 1980.

19. Tanaka H, Zaima N, Sasaki T, Hayasaka T, Goto-Inoue N, Onoue K, et al. Adventitial vasa vasorum arteriosclerosis in abdominal aortic aneurysm. PloS One. 2013;8:e57398.

20. Osada H, Kyogoku M, Ishidou M, Morishima M, Nakajima H. Aortic dissection in the outer third of the media: what is the role of the vasa vasorum in the triggering process? Eur J Cardiothorac Surg. 2013;43:e82-8.

21. Baikoussis NG, Apostolakis EE, Papakonstantinou NA, Siminelakis SN, Arnaoutoglou H, Papadopoulos G, et al. The implication of vasa vasorum in surgical diseases of the aorta. Eur J Cardiothorac Surg. 2011;40:412-7.

22. Buja LM. Clinical anatomy and pathology of the aorta. Evanston: Greenwood Academic; 2009.

23. Evangelista A, Dominguez R, Sebastia C, Salas A, Permanyer-Miralda G, Avegliano G, et al. Long-term follow-up of aortic intramural hematoma: predictors of outcome. Circulation. 2003;108:583-9.

24. Nienaber CA, Kische S, Rousseau H, Eggebrecht H, Rehders T, Kundt G, et al. Endovascular repair of type B aortic dissection: long-term results of the randomized investigation of stent grafts in aortic dissection trial. Circ Cardiovasc Interv. 2013;6:407-16.

25. Tsai TT, Evangelista A, Nienaber CA, Myrmel T, Meinhardt G, Cooper JV, et al. Partial thrombosis of the false lumen in patients with acute type B aortic dissection. N Engl J Med. 2007;357:349-59. 